\title{
On-Off switching properties of one-dimensional photonic crystals consisting of azo-functionalized polymer liquid crystals having different methylene spacers and polyvinyl alcohol.
}

\author{
Ryohei Yagi, ${ }^{\dagger}$ Hideki Katae, ${ }^{\dagger}$ Yutaka Kuwahara, ${ }^{\dagger}$ Sun-Nam Kim, ${ }^{\dagger}$ Tomonari Ogata, \\ and Seiji Kurihara, ${ }^{*}+, \neq, \perp$. \\ ${ }^{\dagger}$ Graduate School of Science and Technology, and ${ }^{\S}$ Innovative Collaboration Organization, Kumamoto \\ University, 2-39-1 Kurokami, Kumamoto 860-8555, Japan \\ †ST, CREST, 7 Gobancho, Chiyoda-ku, Tokyo 102-0076, Japan \\ ${ }^{\perp}$ Kumamoto Institute for Photo-Electro Organics (PHOENICS), 3-11-38 Higashimachi, Higashi-ku, \\ Kumamoto, 862-0901, Japan. \\ Correspondence to: Seiji Kurihara (E-mail: kurihara@gpo.kumamoto-u.ac.jp) \\ ((Additional Supplementary Information may be found in the online version of this article.))
}

\begin{abstract}
In this paper, photoresponsive behavior of multi-bilayered films having precisely controlled layer thickness prepared by stacking an azo-functionalized polymer liquid crystal, PMAzXAc, and polyvinylalcohol alternatively, PVA, is described. The multi-bilayered fims were found to reflect a light of specific wavelength depending on the layer thickness and refractive index, and showed the reversible change in the reflection intensity by irradiation with visible and UV lights. The change in the reflection intensity was brought about by change in the molecular orientation of PMAzXAc between an out-of-plane orientation and a photo-induced isotropic state, and was strongly dependent on the number of methylene spacer of PMAzXAc linking the azobenzene side group with the acrylate polymer main chain. PMAz6Ac with hexa-methylene spacer showed the largest change in the reflection intensity, while smaller change in the reflection intensity was observed for PMAzXAc having shorter or longer methylene spacer than 6 . The effect of the methylene spacers on the photochemical change in the molecular orientation of azobenzene chromophores in the multi-bilayered films will be discussed.

KEYWORDS: azo polymers, liquid-crystalline polymer (LCP), photonic crystals, photoisomerization, reflection

The Photonic crystals exhibit photonic band gap (PBG), and have attracted much attention from both fundamental and practical points of view, because of their unique properties inhibiting the propagation of light due to PBG. ${ }^{1,2,3}$ In particular, multi-bilayered films having precisely controlled layer thickness are known as one-dimensional photonic crystals (1D-PCs). In the case of 1D-PCs consisting of the multi-bilayered structure, both reflection peak wavelength and intensity of reflection depend on the periodicity of layered structure and refractive index of materials. The wavelength of reflection peak, $\lambda$, can be estimated according to Bragg diffraction equation as follows: ${ }^{1,2,4}$
\end{abstract}

$m \lambda=2 a \sqrt{n_{1}^{2} \frac{d_{1}}{a}+n_{2}^{2} \frac{d_{2}}{a}}$ 
where $a$ is the thickness of each bilayer, $n_{1}, n_{2}$ and $d_{1}, d_{2}$ are the refractive indices and the layer thicknesses of stacked materials 1 and 2 in each layer, respectively, and $m$ is the diffraction order integer. So, the wavelength of the reflection can be controlled by varying the thickness of each layer and/or the refractive index of the stacked materials.

In addition, peak reflectance, $R$, of the multi-bilayered films is given by the following equation for the normal incidence of light: ${ }^{5}$

$$
R=\left[\frac{1-\left(n_{H} / n_{L}\right)^{2 q}\left(n_{H}^{2} / n_{S}\right)}{1+\left(n_{H} / n_{L}\right)^{2 q}\left(n_{H}^{2} / n_{S}\right)}\right]^{2}
$$

where $n_{H}$ and $n_{L}$ are the high and low refractive indices of the stacked materials in each bilayer, respectively, $n_{S}$ is the refractive index of the substrate and $q$ is the number of bilayers. From equation (2), the reflectance will be increased with the increase in the difference between $n_{H}$ and $n_{L}$. In contrast the reflectance becomes extremely low when $n_{H}, n_{L}$ and $n_{S}$ are nearly equal. Therefore, an on-off switching of the reflection will be achieved by controlling the difference in the refractive indices of the stacked materials between the equal state $\left(n_{H}=n_{L}=n_{S}\right)$ (FIGURE 1 A) and the different state $\left(n_{H} \neq n_{L}\right)$ (FIGURE 1 B). Recently, we have successfully demonstrated that the on-off switching of the reflection of the multi-bilayered films by switching between the equal and different states ${ }^{6}$. In our previous work, we used polyacrylate having the azobenzene side group via hexamethylene spacer, PMAz6Ac ( $\mathrm{X}=6$ in SCHEME 1) and polyvinyl alcohol (PVA) as the stacked materials. PMAz6Ac was found to show spontaneous out-of-plane orientation in the multi-bilayered films fabricated by alternative spin coating of a PMAz6Ac/cyclohexanone solution and a PVA/water solution on a glass substrate. The multi-bilayered films reflected the light of specific wavelength which was depending on the difference between the refractive indices of PMAz6Ac (1.60, $\mathrm{n}_{\mathrm{av}}$ ) and PVA (1.49) layers, and the bilayer thickness, while the reflection band was disappeared by heating at $80{ }^{\circ} \mathrm{C}$ due to the out-of-plane orientation of PMAz6Ac (FIGURE 1 C), resulting in the decrease in the refractive index of the PMAz6Ac layer $\left(1.48, n_{0}\right)$, which was nearly equal to that of the PVA layer. The reflection band was recovered by irradiation with ultraviolet (UV) light to cause photoisomerization from the trans-form to cis-form, leading to the transformation from the out-of-plane orientation to the photo-induced isotropic state (see supplementary information FIGURE S1) in the PMAz6Ac layer (FIGURE 1 D). In this way, the on-off switching of reflection of the multi-bilayered films was achieved by the combination of thermal self-organization and photochemical disorganization of the azobenzene-functionalized liquid crystalline polymer. 


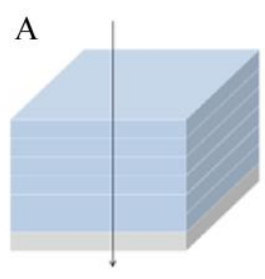

$\mathrm{n}_{\mathrm{H}}=\mathrm{nL}=\mathrm{ns}$

$\mathrm{C}$

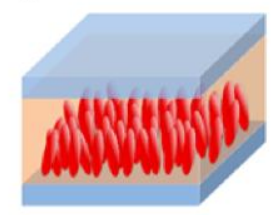

$\mathrm{E}$

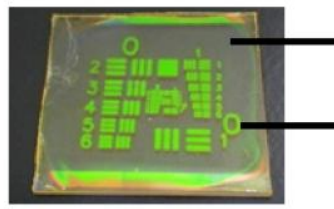

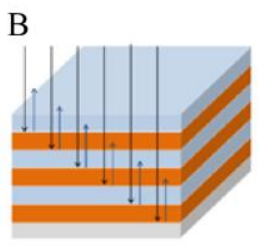

$\mathrm{n}_{\mathrm{H}} \neq \mathrm{nL}$

$\mathrm{D}$

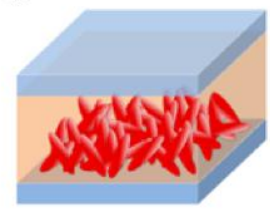

Reflection OFF state

$(\mathrm{nH}=\mathrm{nL}=\mathrm{ns})$

Reflection ON state

$\left(\mathrm{n}_{\mathrm{H}} \neq \mathrm{nL}\right)$

FIGURE 1 Diagram of on-off switching of reflection by changing molecular orientation of azobenzene molecules. (A) On state of reflection of multi-bilayered film $\left(n_{H}=n_{L}=n_{S}\right)$, (B) off state $\left(n_{H} \neq n_{L}\right),(C)$ out-of-plane orientation, (D) random orientation (or photo-induced isotropic state), (E) patterned multi-bilayered film.

On the other hand, it has been reported that variety of molecular orientation can be realized by changing polarization and wavelength of light, consequently the out-of-plane orientation is also produced by irradiation of non-polarized light on an amorphous polymers containing azobenzene groups. ${ }^{7,8}$ If the on-off switching of reflection can be achieved by only irradiation of light without application of thermal energy, such materials will be candidate for the optical devices such as rewritable papers and memories which are completely addressed by optical information. In this work, we investigate the photochemically molecular orientation behavior of the azobenzene-functionalized polyacrylates having different methylene spacer lengths from 3 to 9, and the on-off switching of the reflection of multi-bilayered films consisting of the azobenzene-functionalized polyacrylates and PVA by irradiation with UV and visible (Vis) lights.

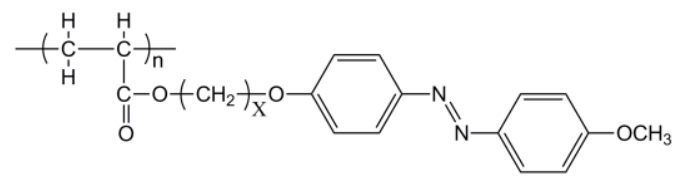

SCHEME 1 Azo-functionalized polymers used in this study, PMAzXAc.

\section{EXPERIMENTAL}

\section{Materials}

Monomers, MAzXAc ( $X=3-9)$ where $X$ is the number of methylene groups in the spacers, were synthesized according to the manner reported earlier ${ }^{9}$ and polymerized in the presence of 2,2-azobisisobutyronitrile (AIBN) as radical initiator in $\mathrm{N}, \mathrm{N}$-dimethylformamide (DMF). The structure and purity of the monomers were identified by NMR, IR and elemental analysis as given in the supporting 
information. Reagent-grade AIBN was recrystallized from methanol, dried under vacuum at room temperature and stored at $-4{ }^{\circ} \mathrm{C}$ before use. DMF was purchased from Wako Pure Chemical Industries Ltd and was pretreated with activated alumina prior to use. Other reagents were used without further purification, unless stated.

Polymerization of the monomers was carried out as follows. First, $2.0 \mathrm{~g}$ of monomer and $20 \mathrm{mg}$ of AIBN were dissolved in $20 \mathrm{ml}$ of DMF in a glass tube. After bubbling argon through the solution, the tube was sealed and shaken in a water bath at $60^{\circ} \mathrm{C}$ for $48 \mathrm{~h}$. The reaction mixture was poured into excess methanol. The precipitate product was collected and dissolved in tetrahydrofuran (THF) again. After repeating this reprecipitation cycle several times, PMAzXAc were collected and dried in vacuum.

\section{Properties of PMAzXAc}

Thermal properties of PMAzXAc were examined by means of differential scanning calorimetry (DSC; Seiko SSC-5020) and polarizing optical microscopy (POM; Olympus BHSP polarizing microscope; Mettler FP90 and FP82 hot stage and controller). DSC measurements were carried out by heating at a rate of 10 ${ }^{\circ} \mathrm{C} / \mathrm{min}$. Molecular weights, $\mathrm{Mn}$ and $\mathrm{Mw}$, were determined by gel permeation chromatography (GPC; Jasco 870-UV detector at $254 \mathrm{~nm}$, Shodex KF-804F column, THF as eluent)

Photoisomerization behaviors of PMAzXAc were studied by monitoring changes in absorption spectra with an UV/Vis spectrometer (Shimazu UV-1600) at room temperature under irradiation of UV or Vis light on their solutions in chloroform and solid films. The solid films of PMAzXAc were prepared on a glass substrate by spin coating method using cyclohexanone as solvent. Thickness of the films was estimated to be about 100 $200 \mathrm{~nm}$ by measuring absorbance. The photoisomerization of azo-chromphores between trans- and cis-form was carried out by using a 500-W high pressure mercury lamp (Ushio SX-UI 5000) equipped with an adequate cut filter (Sigma UTVAF-36U or Sigma SCF-50S-42L) for UV(365 $\mathrm{nm})$ or Vis $(436 \mathrm{~nm})$ light irradiation.

Multi-bilayered films were fabricated by alternative spin coating of PMAzXAc and PVA solutions on a glass substrate $(25 \times 20 \mathrm{~mm})$. Spin coating was typically performed as follows: spin rate of $3000 \mathrm{rpm}$ with $4.5 \mathrm{wt} \%$ of PMAz6Ac in cyclohexanone and $2.5 \mathrm{wt} \%$ of PVA in water for providing the multi-bilayered film showing reflection band at around $580 \mathrm{~nm}$ (PMAz6Ac: ca. $95 \mathrm{~nm}$, PVA: ca. $90 \mathrm{~nm}$ ). After spin coating each layer was dried for $10 \mathrm{~min}$ at room temperature. The reflection spectra of the multi-bilayered films were measured with a spectrometer (Ocean optics USB2000) with or without irradiation of UV and/or Vis light with a 500-W high pressure mercury lamp.

\section{RESULTS AND DISCUSSION}

Polyacrylates having azobenzene groups, PMAzXAc, were synthesized by radical polymerization. Molecular weights, polydispersities and thermal properties of PMAzXAc are given in TABLE 1. The polymers having methylene spacers from 5 to 9 exhibit both nematic and smectic phases as liquid crystalline phases, while only the nematic phase was observed for PMAz3Ac and PMAz4Ac (see supplementary information, FIGURE S2). 
TABLE 1 Molecular weights and phase transition temperatures of PMAzXAc.

\begin{tabular}{cccccccccc}
\hline Sample & Mn & Mw/Mn & \multicolumn{5}{c}{ Thermal properties $\left({ }^{\circ} \mathrm{C}\right)$} \\
\hline PMAz3Ac & 5300 & 1.24 & G & 50 & & N & 99 & I \\
PMAz4Ac & 6300 & 1.34 & G & 48 & & & N & 123 & I \\
PMAz5Ac & 6500 & 1.34 & G & 55 & S & 81 & N & 119 & I \\
PMAz6Ac & 6400 & 1.35 & G & 64 & S & 95 & N & 135 & I \\
PMAz7Ac & 8400 & 1.46 & G & 60 & S & 107 & N & 121 & I \\
PMAz8Ac & 7200 & 1.36 & G & 50 & S & 117 & N & 130 & I \\
PMAz9Ac & 8900 & 1.42 & G & 52 & S & 96 & N & 136 & I \\
\hline
\end{tabular}

$\mathrm{G}, \mathrm{S}, \mathrm{N}$ and I represent glassy, smectic, nematic and isotropic phases, respectively

Photoisomerization behaviors of PMAzXAc were studied in solution and solid film. Films of PMAzXAc were prepared by spin coating of their solutions in cyclohexanone onto cleaned glass substrate. The spin-coated films were air dried at room temperature. Film thickness was controlled by adjusting the concentration of solutions and speed of spin coating. Photoisomerization of the PMAzXAc solutions and films were carried out by using the 500-W high pressure mercury lamp. FIGURE 2 shows the changes in absorption spectra of PMAz6Ac in chloroform (A, B) and in film (C, D) by irradiation with UV and Vis lights. The absorption spectra are characterized by a strong $\pi-\pi^{*}$ transition of trans-azobenzene at around $360 \mathrm{~nm}$ and a weak absorption at around $450 \mathrm{~nm}$, which originates from $\mathrm{n}-\pi^{*}$ transition. Upon UV irradiation $\left(365 \mathrm{~nm}, 10 \mathrm{~mW} / \mathrm{cm}^{2}\right.$ ), absorbance at $360 \mathrm{~nm}$ decreased and absorbance at $450 \mathrm{~nm}$ increased slightly due to trans-to-cis photoisomerization. Conversely, Vis light irradiation (436 nm, 58 $\mathrm{mW} / \mathrm{cm}^{2}$ ) caused a reverse situation to be observed. Thus, UV irradiation causes a photoisomerization from the trans-form to the cis-form, while Vis irradiation reversibly recovers the trans-form. Interestingly, in addition, the absorbance of the PMAz6Ac film after Vis light irradiation was higher than the initial absorbance of the fresh PMAz6Ac film. This is due to the out-of-plane orientation of azobenzene chromophores of PMAz6Ac in the fresh film as discussed later.
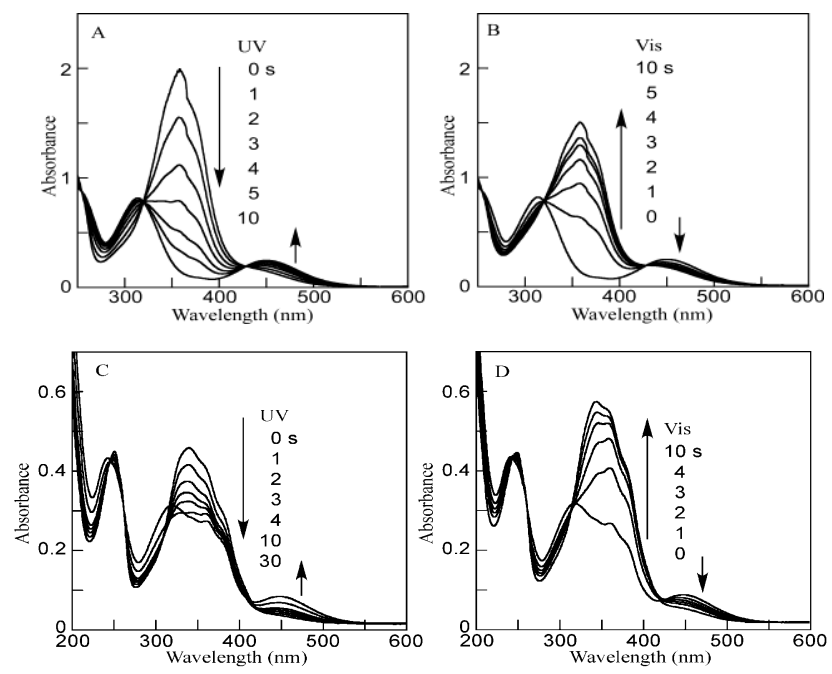

FIGURE 2 Changes in absorption spectra of PMAz6Ac in chloroform (A, B) and solid films (C, D) by irradiation with UV light $\left(365 \mathrm{~nm}, 10 \mathrm{~mW} / \mathrm{cm}^{2}\right)(A, C)$ and following visible light $\left(436 \mathrm{~nm}, 58 \mathrm{~mW} / \mathrm{cm}^{2}\right)(B$, D). 
FIGURE 3 shows the absorption spectra of PMAzXAc (X=3, 6 and 8) in chloroform and fresh films prepared on a quartz substrate. It is reported that the relative intensity of the $\pi-\pi^{*}$ absorption band and the $\phi-\phi^{*}$ absorption band at around $250 \mathrm{~nm}$ is related to the formation of the out-of-plane orientation of the azobenzene chromophores in polymer liquid crystals. ${ }^{10}$ The relative intensity of three polymers in solution remained almost the same, while significant change in the relative intensity was observed in the films, indicating the influence of the methylene spacer on the formation of the out-of-plane orientation of the azobenzene chromophores in the films. So, in order to clarify the effect of the spacer length on the molecular orientation of the azobenzene chromophores in a solid film, the relative intensity of absorbance at $250 \mathrm{~nm}$ to that at $350 \mathrm{~nm}, A_{250} / A_{350}$, is plotted as a function of the methylene spacer length, $X$. It is apparent from FIGURE 4 that $A_{250} / A_{350}$ of the solid films is increased with the increase in the spacer length. The azobenzene chromophores in the polymers having longer methylene spacers tend to be in the out-of-plane orientation in a fresh film. These results indicate that the increase in the absorbance corresponding to the $\pi-\pi^{*}$ transition by UV and following Vis light irradiation on the PMAz6Ac film (FIGURES 2C and D) is related to the out-of-plane orientation of the azobenzene chromophores in the PMAz6Ac fresh film. Although the absorbance was depressed due to the out-of-plane orientation in the fresh film, disorganization of the out-of-plane orientation to random orientation caused the increase in the absorbance through the reversible trans-cis photoisomerization of azobenzene chromophores.
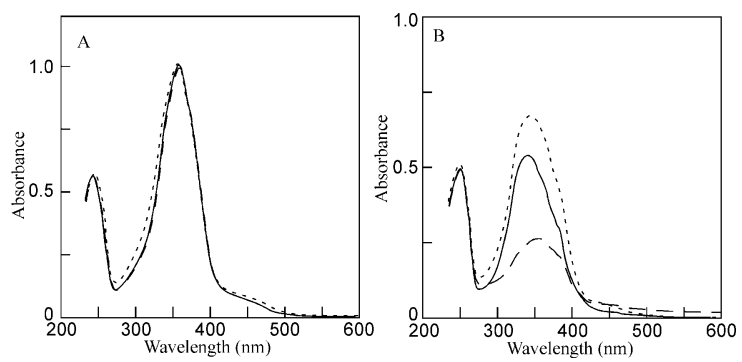

FIGURE 3 Absorption spectra of PMAz3Ac (dotted line), PMAz6Ac (solid line) and PMAz8Ac (dashed line) in chloroform (A) and solid film (B) on a quartz substrate.

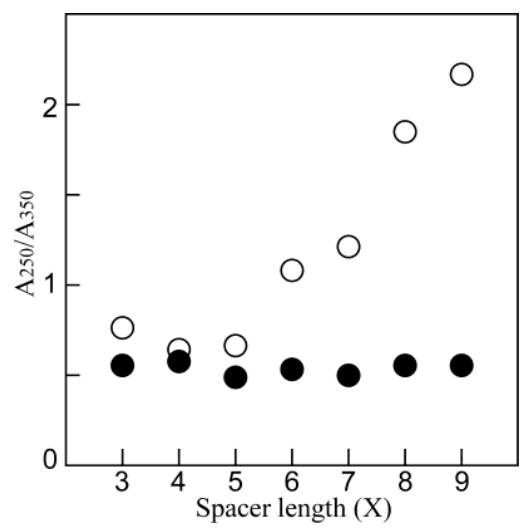

FIGURE 4 Effect of the spacer length on the molecular orientation of PMAzXAc in chloroform (closed circle) and in a solid film (open circle). 
As we have mentioned in our previous study, PMAz6Ac was found to exhibit the transformation from the random orientation to the out-of-plane orientation not only in the solid film prepared on a glass substrate, but also in a multi-bilayered film consisting of PMAz6Ac and PVA by annealing in the smectic phase. ${ }^{6}$ However, the out-of-plane orientation of azobenzene chromophores can also be produced by irradiation with non-polarized light of appropriate wavelength to cause continuous trans-cis-trans isomerization cycle., ${ }^{7,8}$ Prior to explore the photochemical on-off switching of reflection of multi-bilayered films consisting of PMAzXAc and PVA as the stacked materials, the transformation behavior from the random orientation to the out-of-plane orientation by thermal annealing $\left(70-90{ }^{\circ} \mathrm{C}\right.$, $10 \mathrm{~min}$ ) or irradiation with non-polarized Vis light (436 nm, $58 \mathrm{~mW} / \mathrm{cm}^{2}, 30 \mathrm{~min}$ ) was clarified. In order to discuss the effect of the methylene spacers on the out-of-plane orientation, the order parameter, $\mathrm{S}$, of the out-of-plane orientation was estimated according to the manner reported previously. ${ }^{6,10}$ FIGURE $5 \mathrm{~A}$ shows change in the absorption spectra of the PMAz6Ac film upon annealing at the smectic phase $\left(80^{\circ} \mathrm{C}\right)$ for $10 \mathrm{~min}$. Upon annealing, the absorbance at $350 \mathrm{~nm}$ was significantly decreased by a factor of around 5. Polarized absorption spectra of the film at an incident angle of $45^{\circ}$ to the normal of the film were measured by rotating the polarization plane of the monitor light (FIGURE 5C). As shown in FIGURE $5 B$, the $\pi-\pi^{*}$ absorption band at the rotation angle of $0^{\circ}$ was shifted to shorter wavelength (curve $A_{0}$ ), whereas at $90^{\circ}$ a weak $\pi-\pi^{*}$ band was observed (curve $A_{90}$ ). This result revealed that the annealing at 80 ${ }^{\circ} \mathrm{C}$ contributed not only to the formation of the out-of-plane orientation (supplementary information FIGURE S1), but also to the formation of $\mathrm{H}$-aggregates of the azobenzene chromophores. ${ }^{8}$

Here, the order parameter, $\mathrm{S}$, of the out-of-plane orientation was determined with the following equation by using the absorbances, $A_{0}$ and $A_{90}{ }^{6,10}$

$$
S=\frac{A_{0}-A_{90}}{A_{0}+2 A_{90}}
$$



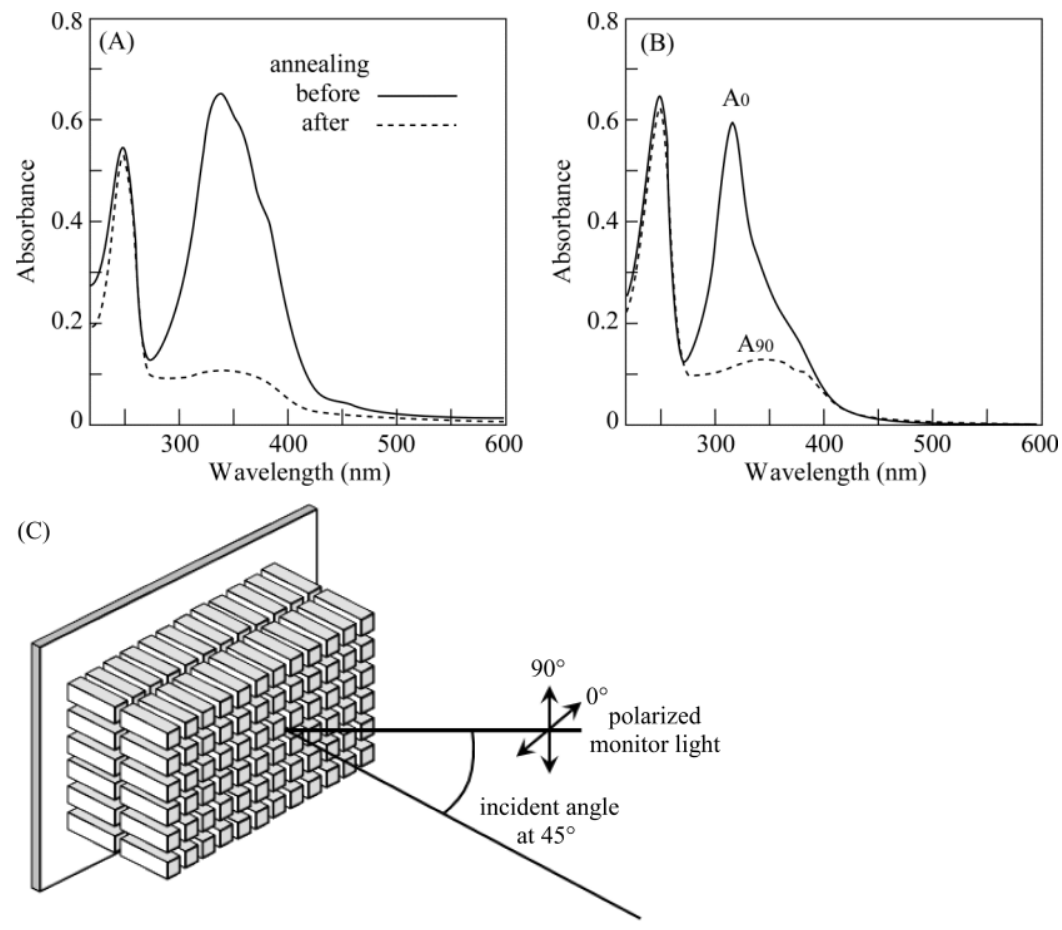

FIGURE 5 Absorption spectra of the PMAz6Ac film before and after annealing (solid and dotted lines) (A) and change in the polarized absorption spectra at an incident angle of $45^{\circ}$ to the normal of the film by rotating the polarization plane of the monitor light $\left(A_{0}\right.$ and $A_{90}$ are spectra at the rotation angle of $0^{\circ}$ and $90^{\circ}$, respectively) (B), (C): the rotational angle for the measurements of polarized absorption spectra.

In the case of the thermal annealing, the highest order parameter of the out-of-plane orientation obtained in the temperature range studied for each polymer was plotted as a function of methylene spacer length in FIGURE 6.

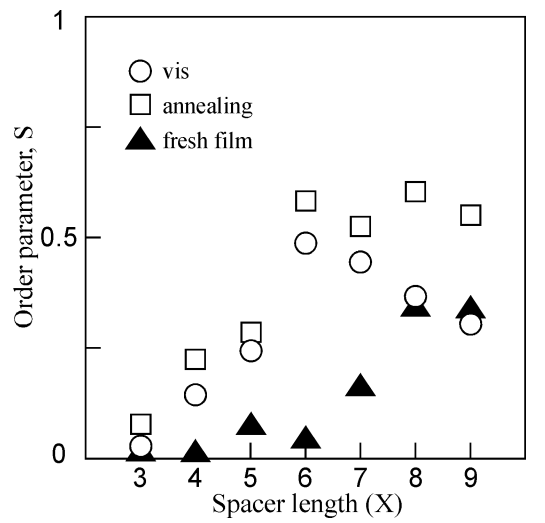

FIGURE 6 Influence of spacer length on the out-of-plane molecular orientation of PMAz6Ac layer by non-polarized visible light irradiation (circle, $436 \mathrm{~nm}, 140 \mathrm{~mW} / \mathrm{cm}^{2}, 30 \mathrm{~min}$ ) and heating in the smectic phase (square, $80-90^{\circ} \mathrm{C}$ ).

The polymers having methylene spacers in the range of 6 to 9 are found to show higher order parameters after annealing (square in FIGURE 6) compared to the polymers having shorter spacers. 
There are a lot of papers on the photoinduced birefringence via the photoinduced motion of azobenzene chromophores linked polymer chains. ${ }^{7}$ These results have suggested that the shorter spacers restrict the motion of azobenzene groups, leading to lower photoinduced birefringence. ${ }^{11}$ Therefore, it is likely that the motion and flexibility of side chain groups are contributors to the formation of out-of-plane orientation, consequently the order parameter increases with the increase in the methylene spacer length. Contrary to the annealing, the decrease in the order parameter was observed for PMAzXAc having longer spacers $(X=7-9)$ by irradiation with non-polarized Vis light at room temperature. It has also been reported that significant decrease in the photochemically induced molecular motion was recognized in the smectic phase. ${ }^{11}$ Although no distinct smectic phase structure was recognized in the fresh PMAZXAc $(X=7-9)$ films based on the $X$-ray diffraction measurements, higher out-of-plane orientation was observed in the fresh films of the polymers $(X=7-9)$ at room temperature compared to other polymers $(X=3-6)$ as shown in FIGURES 4 and 6 . In addition, the blue shift in the polarized absorption spectra of the fresh films reveals that PMAzXAc $(X=7-9)$ tend to form $\mathrm{H}$-aggregate of the azobenzene chromophores. These data indicate that the motion of azobenzene chromophores in the PMAzXAC $(X=7-9)$ under photoirradiation is lower than other polymers at room temperature, ${ }^{8}$ contributing to lower order parameter of the out-of-plane orientation of the polymers with longer spacer lengths.

The multi-bilayered films having precisely controlled layer thickness can reflect the light of specific wavelength depending on the thickness and refractive index of the stacked layers according to equation (1). On the other hand, peak reflectance, $R$, of the multi-bilayered films is increased with the increase in the number of stacked bilayers according to equation (2). Actually, the intensity and wavelength of reflection of the multi-bilayered films consisiting of PMAz6Ac and PVA are dependent on the number of bilayers and the layer thickness (supplementary information, FIGURES S3 and S4), respectively. The multi-bilayered films were fabricated by alternative spin coating of PMAz6Ac/cyclohexanone and PVA aqueous solutions on a glass substrate. FIGURE 7 shows the change in the reflection spectra of the PMAz6Ac/PVA multi-bilayered film by thermal annealing and irradiation with UV light. The number of the stacked bilayers consisting of PMAz6Ac and PVA was 20, and the optical thickness ratio of the PMAz6Ac and PVA layers was adjusted to be roughly a quarter of a reflection wavelength, because the reflectance is also dependent on the optical thickness of the stacked layers (supplementary information, ratio of 1:1 in FIGURE S5). ${ }^{12}$ The reflectance was decreased and increased by the annealing at the smectic phase and following UV irradiation, respectively. This reversible change in the reflectance is related to the thermal out-of-plane orientation and photochemical disorganization effect of the azobenzene group, resulting in the transformation between the out-of-plane orientation and photo-induced isotropic state, as we reported previously. ${ }^{6}$

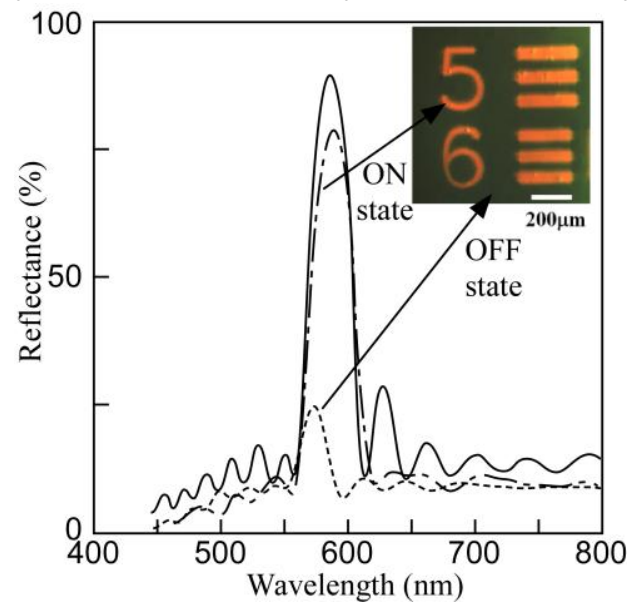


FIGURE 7 Change in reflection spectra of the PMAz6Ac/PVA 20-multibilayered film by annealing at $80{ }^{\circ} \mathrm{C}$ for $10 \mathrm{~min}$, and following irradiation with UV light $\left(365 \mathrm{~nm}, 40 \mathrm{~mW} / \mathrm{cm}^{2}\right)$ for $10 \mathrm{~min}$, and patterned film (inserted figure). (solid line) Before annealing, (dotted line) after annealing, (dash-dot line) after annealing and UV irradiation.

Here, to explore the orientational behavior of the azobenzene group in the multi-bilayered films, the change in the reflectance of the PMAz6Ac/PVA 20-multi-bilayered film was measured by annealing at the smectic phase $\left(80^{\circ} \mathrm{C}\right)$ or irradiation with non-polarized Vis light $\left(436 \mathrm{~nm}, 140 \mathrm{~mW} / \mathrm{cm}^{2}\right)$ as given in FIGURE 8. The ordinary and extraordinary refractive indices of PMAz6Ac, $n_{0}$ and $n_{e}$, are estimated to be 1.48 and 1.81 respectively by using wedge cell. ${ }^{13}$ The apparent initial reflectance of the fresh film was about $90 \%$, since the difference in the refractive indices between the PMAz6Ac layer where the azobenzene chromophores were in the random orientation $\left(n_{\mathrm{av}}\right)$ and the PVA layer $\left(n_{\mathrm{PVA}}\right): \mathrm{n}_{\mathrm{av}}$ and $\mathrm{n}_{\text {PVA }}$ are 1.60 and 1.49, respectively. The reflectance was decreased by the annealing as well as the light irradiation, and became plateau, indicating the transformation from the random orientation to the out-of-plane orientation. Based on equation (2), the initial reflectance $\left(n_{a v}\right.$ and $n_{P V A}=1.60$ and 1.49) and final reflectance $\left(n_{a v}\right.$ and $n_{P V A}=1.48$ and 1.49 ) should be roughly 87 and less than $1 \%$, respectively: However, the refractive index of the PMAz6Ac layer is estimated to be respectively around 1.505 and 1.515 after the irradiation and annealing at the final state. This result reveals that the order of the out-of-plane orientation induced by the annealing is less than that induced photochemically, contrary to the case of the single polymer layer on the glass substrate (FIGURE 6). The less out-of-plane orientation induced by the annealing is attributed to the properties of the interface of PMAz6Ac layer, maybe hydrophobicity (or hydrophilicity). The order of the out-of-plane orientation of the PMAz6Ac layer which is sandwiched between PVA layers, PVA/ PMAz6Ac/PVA/glass film, is significantly less than other films, PMAz6Ac/PVA/glass and PMAz6Ac/glass films (supplementary information, FIGURE S6).

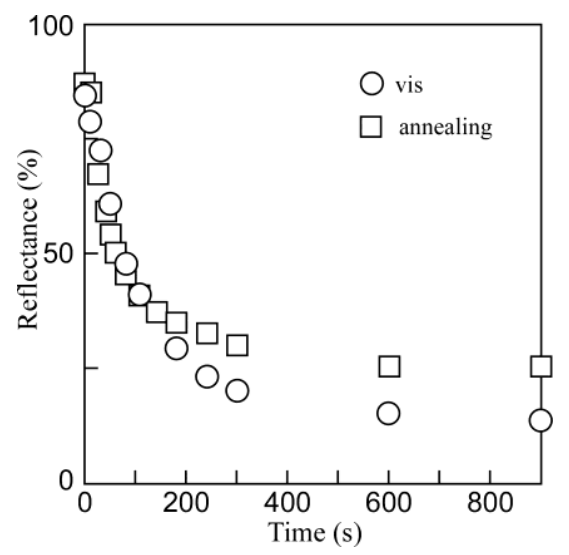

FIGURE 8 Time courses of change in maximum reflectance of the PMAz6Ac/PVA 20-multibilayered films by non-polarized visible light irradiation (circle: $436 \mathrm{~nm}, 140 \mathrm{~mW} / \mathrm{cm}^{2}$ ) and heating in the smectic phase (square, $80^{\circ} \mathrm{C}$ ).

It can be assumed from these results that the change in the reflectance by a light irradiation is favorable compared to the use of the annealing for stable on-off switching of the reflection of multi-bilayered films. So, the effect of the spacer length on the photochemical on-off switching of the reflection was clarified by using 20-bilayered films consisting of PMAZXAc $(X=3-8)$ and PVA, because clear 20-bilayered film containing PMAz9Ac could not be obtained because of low solubility as well as higher associative property. FIGURE 9 shows the changes in the reflectance of the 20-bilayered films by irradiation with non-polarized Vis light $\left(436 \mathrm{~nm}, 140 \mathrm{~mW} / \mathrm{cm}^{2}, 15 \mathrm{~min}\right.$ ) and following UV light (365 nm, $40 \mathrm{~mW} / \mathrm{cm}^{2}, 15$ 
min). Three types of behavior are observed as a function of the spacer length. The fresh films containing PMAzXAc having shorter spacer lengths $(X=3-5)$ as the stacked material exhibited higher reflectance more than others, and the change in the reflectance by irradiation with non-polarized Vis/UV lights was small. Contrary to these films, the reflectance of the fresh PMAz8Ac/PVA 20-bilayered film was extremely low, because of highly out-of-plane orientation of the PMAz8Ac layer even in the multi-bilayered film. Then, the change in the reflectance of the PMAz8Ac/PVA 20-bilayered film was small by irradiation with non-polarized Vis/UV lights. However, the PMAzXAc $(X=6,7) / P V A$ 20-bilayered films were found to show significant large change in the reflectance by non-polarized Vis/UV light irradiation, arising from the effective reversible change in the molecular orientation between the photo-induced isotropic state and out-of-plane states.

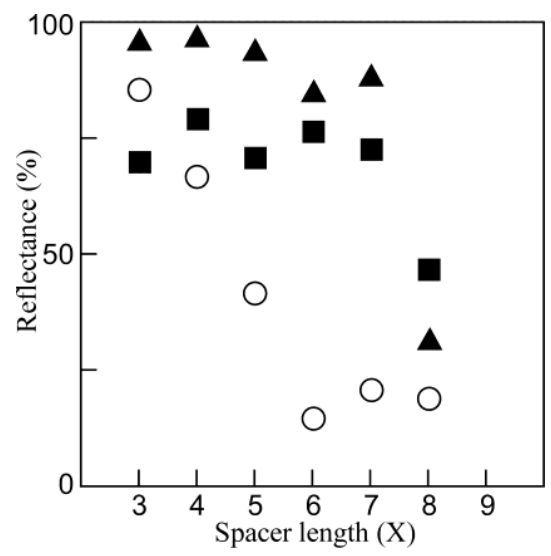

FIGURE 9 Change in reflectance of the PMAzXAc (X=3-8)/PVA 20-multibilayered film by irradiation with non-polarized visible light $\left(436 \mathrm{~nm}, 140 \mathrm{~mW} / \mathrm{cm}^{2}, 15 \mathrm{~min}\right.$ ) and following UV light $\left(365 \mathrm{~nm}, 40 \mathrm{~mW} / \mathrm{cm}^{2}\right.$, 15min). (closed triangle): Fresh films, (open circle): non-polarized visible light, (closed square): non-polarized visible light and UV light.

To clarify the effect of the spacer length on the photochemical on-off switching of the reflection, the changes in the photochemical refractive index of the PMAzXAc layers, $\Delta n_{\text {vis/Uv }}$, were estimated according to equation (2) with reflectances given in FIGURE 9, and are given as a function of the spacer length in FIGURE 10.

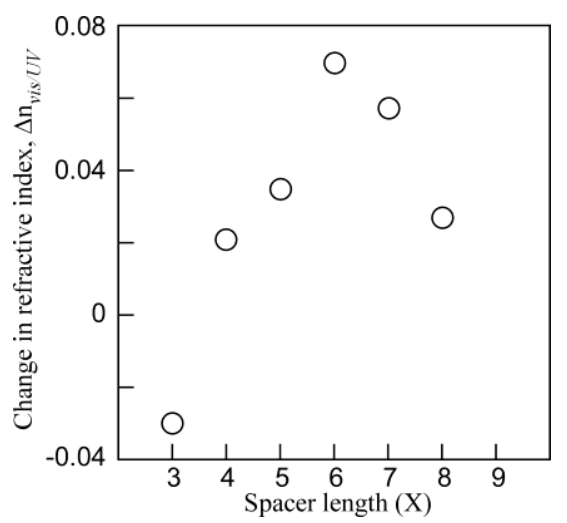

FIGURE 10 Change in refractive index of the PMAzXAc (X=3-8) layers by irradiation with UV light (365 $\left.\mathrm{nm}, 40 \mathrm{~mW} / \mathrm{cm}^{2}, 15 \mathrm{~min}\right)$ after irradiation with non-polarized visible light $\left(436 \mathrm{~nm}, 140 \mathrm{~mW} / \mathrm{cm}^{2}, 15\right.$ $\mathrm{min})$. The refractive indices before and after irradiation with UV light were estimated according to equation (2). 
Most of the films showed the increase in the refractive index (positive $\Delta n_{\text {vis/uv }}$ ) by UV irradiation due to the transformation from the out-of-plane orientation to the photo-induced isotropic state because of the disorganizing effect of the cis-azobenzene, whereas only the PMAz3Ac layer was found to exhibit the

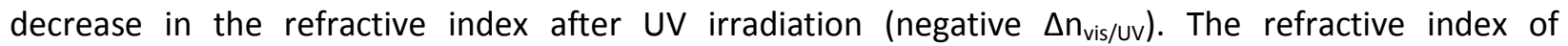
cis-azobenzene of PMAz6Ac could be estimated to be 1.48 by using wedge glass cell. ${ }^{13}$ Although the refractive index of the cis-azobenzene chromophores of PMAz3Ac was not determined experimentally, it must be nearly equal to PMAz6Ac, becuase both polymers possess the same azochromophores in the side groups. Therefore the decrease in the refractive index depending on the content of cis-azobenzene contributes to the negative $\Delta n_{\text {vis/Uv }}$ for the PMAz3Ac/PVA 20-bilayered film. In addition, $\Delta n_{\text {vis/Uv }}$ was increased with the increase in the spacer length up to 6 , and PMAz6Ac showed the highest $\Delta \mathrm{n}_{\text {vis/uv. This }}$ is related to the effective change in the molecular orientation between the out-of-plane orientation and the photo-induced isotropic state under UV light irradiation. However, $\Delta \mathrm{n}_{\text {vis/Uv }}$ was decreased by the increase in the spacer length of PMAzXAc longer than 6. The depression of molecular motion due to stronger molecular interaction between azobenzene chromophores having longer spacers results in the less response to the disorganizing effect of the cis-azobenzene chromophores.

\section{CONCLUSIONS}

Polyacrylates, PMAzXAc, having different methylene spacers between the polyacrylate main chain and the azobenzene side group from 3 to 9 were synthesized and the effect of the methylene spacer length on the reversible on-off switching of reflection of the multi-bilayered films consisting of PMAzXAc and PVA was investigated. The switching behavior of reflection was closely related to the thermal or photochemical change in the molecular orientation between the photo-induced isotropic state and out-of-plane orientation. In this study, PMAz6Ac showed the highest change in the reflection intensity by Vis/UV light irradiation. Stable switching was repeatedly achieved by irradiation with Vis/UV light as shown in FIGURE 11. The multi-bilayered films can be fabricated on a flexible film as shown in FIGURE S7. Therefore, the multi-bilayered films showing the on-off switching of reflection intensity will be candidate as recording materials such as a substitute material for paper.

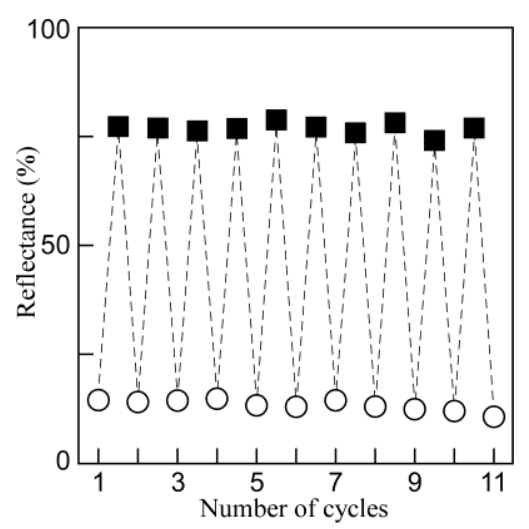

FIGURE 11 Repetition property of change in reflectance of the PMAz6Ac /PVA 20-multibilayered film by irradiation with non-polarized visible light $\left(436 \mathrm{~nm}, 140 \mathrm{~mW} / \mathrm{cm}^{2}, 15 \mathrm{~min}\right.$, open circle) and UV light (365 $\mathrm{nm}, 40 \mathrm{~mW} / \mathrm{cm}^{2}, 15 \mathrm{~min}$, closed square).

\section{ACKNOWLEDGEMENTS}

This work was supported by JSPS KAKENHI Grant 23350115, and the Core Research for Evolutional Science and Technology (CREST) of the Japan Science and Technology Agency (JST). 


\section{REFERENCES AND NOTES}

1. E. Yablonovitch, Phys. Rev. Lett., 1987, 58, 2059-2062.

2. S. John, Phys. Rev. Lett., 1987, 58, 2486-2489.

3. K. Busch, S. Lölkes, R.B. Wehrspohn and H. Föll, Photonic Crystals, WILEY-VCH, 2004.

4. S. Kinoshita, S. Yoshioka and J. Miyazaki, Rep. Prog. Phys. 2008, DOI : 10.1088 /0034-4885/71/7/076401

5. T. Komikado, A. Inoue, K. Masuda, T. Ando and S. Umegaki, Thin Solid Films, 2007, 515, 3887-3892.

6. M. Moritsugu, T. Ishikawa, T. Kawata, T. Ogata, Y. Kuwahara and S. Kurihara, Macromol. Rapid Comm., 2011, 32, 1546-1550.

7. A. Natansohn and P. Rochon, Chem. Rev., 2002, 102, 4139-4175.

8. M. Han and K. Ichimura, Macromolecules, 2001, 34, 82-89.

9. T. Ikeda, S. Horiuchi, D.B. Karanjit, S. Kurihara and S. Tazuke, Macromolecules, 1990, 23, 36-42.

10. A. Bobrovsky, N. Boiko, V. Shibaev and J. Stumpe, J. Photochem. Photobiol. A: Chem., 2004, 163, 347-358.

11. F. Labarthet, S. Freiberg, C. Pellerin, M. Pezolet, A. Natansohn and P. Rochon, Macromolecules, 2000, 33, 6815-6823.

12. T. Komikado, S. Yoshida and S. Umegaki, Appl. Phys. Lett., 2006, 83, 061123 1-3.

13. A. Fukuda and H. Takezoe, Structures and Properties of Ferroelectric liquid crystals (in japanese). Corona Pub. Co., Ltd., : Tokyo, 1990. 


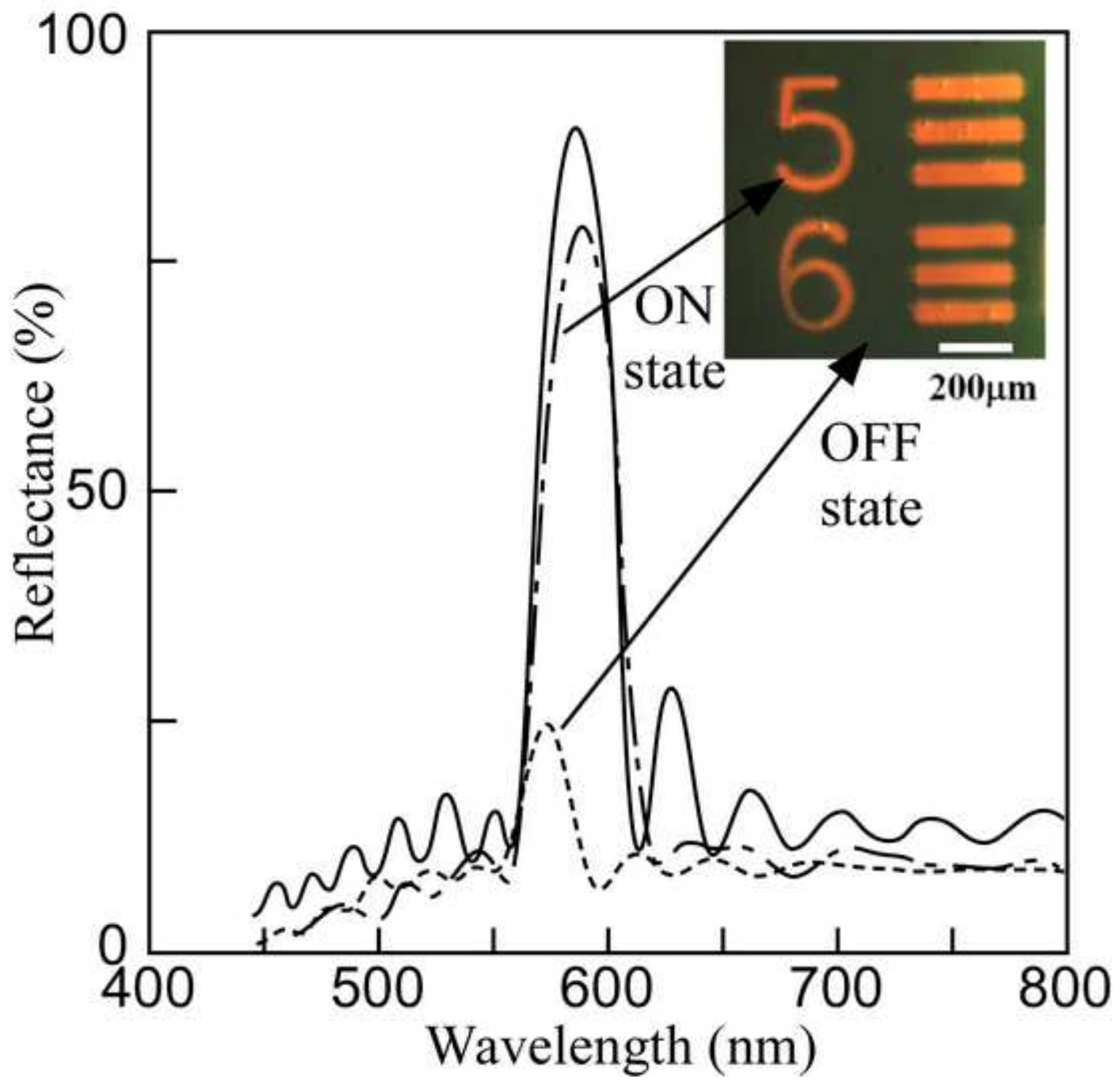

